\title{
Dolor musculoesquelético en fisioterapeutas pediátricos
}

\author{
Musculoskeletal pain in pediatric physiotherapists \\ Liseth A. Arellano Muguruza ${ }^{1}$, Lucía J. Oropeza Melgar ${ }^{1}$,Lupe Vidal Valenzuela ${ }^{1}$, Teofilo Camacho \\ Conchucos ${ }^{2,3}$
}

\section{RESUMEN}

El ejercicio profesional del fisioterapeuta dentro de su área laboral está caracterizado por realizar movimientos repetitivos, cargas y descargas de peso, el cual si no se realiza de una manera adecuada tendrá como consecuencias alteraciones músculo-esqueléticas que causan dolor. Objetivo: Determinar la frecuencia del dolor músculoesquelético en fisioterapeutas pediátricos en instituciones de salud de la ciudad de Lima. Material y Métodos: Se realizó un estudio de tipo observacional - descriptivo de corte transversal. El grupo de estudio estuvo constituido por 47 fisioterapeutas pediátricos de dos instituciones de salud ubicadas en la ciudad de Lima. Para recolectar la información se utilizó la encuesta como técnica y como instrumento se utilizó un cuestionario sobre el dolor músculo-esquelético elaborado para este fin. Resultados: El 100\% de los fisioterapeutas pediátricos presentaron dolor y el $95,7 \%$ en los últimos 12 meses. El área más afectada fue el cuello $(78,7 \%)$. La edad promedio es de 30,3 años, y los años de experiencia fueron 6,1 $\pm 5,7$ años, rango de 1 a 24 años. La principal medida preventiva que los fisioterapeutas encuestados, siempre realizan durante su campode trabajo es utilizar diferentes partes de su cuerpo para realizar alguna técnica fisioterapéutica durante el tratamiento. Conclusiones: La presencia de dolor musculoesqueléticos en fisioterapeutas pediátricos en los últimos 12 meses fue de 95,7\% y en los últimos 7 días $89,4 \%$. A pesar de conocer las estrategias y las medidas preventivas observamos que los fisioterapeutas no lo realizan con frecuencia.

PALABRAS CLAVE: Fisioterapeutas pediátricos, dolor musculoesqueléticos, estrategias de prevención.

\section{SUMMARY}

The physical exercise of the physiotherapist within his / her work area is characterized by repetitive movements, loads and weight discharges, which, if not performed properly, will result in musculoskeletal disorders causing pain. Objective: To determine the frequency of musculoskeletal pain in pediatric physical therapists of health institutions in the city of Lima. Material and Methods: A descriptive observational cross-sectional study will be carried out to 47 pediatric physical therapists from two health institutions located in the city of Lima. To collect the information, the survey will be used as a technique and a questionnaire on musculoskeletal pain will be used as instrument. Results: $100 \%$ of pediatric physiotherapists presented pain and $95.7 \%$ in the last 12 months. The most an affected area was the neck (78.7\%). The average age is 30.3 years, and the years of experience was $6.1 \pm 5.7$ years, range from $1-24$ years. The main preventive measure that the physiotherapists surveyed, always perform during their field of work is to use different parts of their body to perform some physiotherapeutic technique during the treatment. Conclusion: The presence of musculoskeletal pain in pediatric physiotherapists in the last 12 months was $95.7 \%$ and in the last

\footnotetext{
Universidad Peruana Cayetano Heredia. Lima, Perú.

Universidad Nacional Mayor de San Marcos. Lima, Perú.

Instituto Nacional De Rehabilitacion "Dra. Adriana Rebaza Flores” Amistad Peru - Japon. Lima, Perú.
} 
7 days $89.4 \%$. Despite knowing the strategies and preventive measures we observed that the physiotherapists do not perform it frequently.

KEYWORDS: Pediatric physiotherapists, musculoskeletal pain, prevention strategies.

\section{INTRODUCCIÓN}

La carrera del fisioterapeuta se caracteriza por buscar el desarrollo adecuado de las funciones que producen los sistemas del cuerpo, donde su buen o mal funcionamiento, repercute en la cinética o movimiento corporal humano. Según la OMS el fisioterapeuta en su campo laboral ejecutará pruebas manuales para determinar el valor de la afectación y fuerza muscular, pruebas para determinar las capacidades funcionales, la amplitud del movimiento articular y medidas de la capacidad vital, así como ayudas diagnósticas para el control de la evolución (1).

Por lo tanto el objetivo laboral de un fisioterapeuta estará basado en la corrección física y en la prevención de las alteraciones del movimiento cinético del hombre, mejorando su calidad de vida para la inclusión social. Sin embargo, a pesar que el fisioterapeuta busca corregir las alteraciones músculo-esqueléticas y a la vez calmar el dolor, su condición laboral y los tipos de inadecuadas posturas le van a generar dolor músculo esquelético.

Este riesgo laboral como en toda profesión se dará como consecuencia de los esfuerzos prolongados, movimientos repetitivos, la inadecuada forma de manipular cargas, levantamientos frecuentes $\mathrm{o}$ pesados, empujar, jalar, trasladar, posturas inadecuadas y prolongadas sumadas a la edad y los años de trabajo. A nivel de formación profesional en el plan curricular de la profesión, no todos son formados con un conocimiento ergonómico adecuado para ejercer su labor, generando que muchos de ellos no tomen en cuenta los riesgos que causan las posturas inadecuadas para desarrollar su trabajo, llevándolos a tener como consecuencia lesiones músculo-esqueléticas que generan dolor.

A nivel de riesgo laboral del fisioterapeuta se encontró que a mayor frecuencia de actividades repetitivas y sumadas a la presencia de fatiga muscular durante la jornada, hay más probabilidad de aparición de dolor músculo esquelética sobre todo si no se realiza con un buen mantenimiento físico de fuerza, coordinación, flexibilidad e higiene postural (2-
6). Según algunos estudios esta dolencia en la actividad profesional de los fisioterapeutas ha empeorado llevándolos a un ausentismo laboral debido a que el dolor, por causa de una lesión músculo-esquelética los ha incapacitado temporalmente o permanentemente para realizar sus tareas laborales (7).

La afectación del sistema musculoesquelético es un problema de salud pública a nivel internacional desde años atrás, así lo demuestra una publicación de la Revista Española de Rehabilitación en 1995 (8) donde se menciona que el ausentismo de profesionales de salud en general en esta época, debido a lesiones musculoesqueléticas fue por lumbalgias, dorsopatias, síndrome del túnel del carpo, tendinopatias. De la misma forma otra publicación menciona de los desórdenes musculoesqueléticos que están entre los problemas médicos más frecuentes en el año 1999 representó la primera causa de ausentismo laboral en EEUU, con un costo anual de 13 billones de dólares. En Japón y Canadá en el año 1998 constituyeron la primera causa de morbilidad ocupacional (9). En Venezuela, el Departamento de Medicina Industrial del Instituto Venezolano del Seguro Social (IVSS) señala que durante los años 1994 - 1998 ocuparon el quinto lugar dentro de las enfermedades profesionales, así mismo la Dirección de Medicina del Trabajo del IVSS determinó que en el período 1999 - 2002 las lesiones músculo-esqueléticas ocuparon el primer lugar dentro de la estadística nacional de Enfermedades Ocupacionales (10).

Las posturas de trabajo muy exigentes por tiempos prolongados como: bipedestación con flexión o torsión constante de tronco, levantamiento constante de cargas ligeras o pesadas, generan lesiones músculo esqueléticas que causan dolor músculo esquelético en varias zonas del cuerpo como: cuello, hombro, muñeca, mano, columna, cadera, rodilla, tobillo y pie. Merskey define al dolor como una experiencia sensorial y emocional, generalmente desagradable. Es una experiencia que experimentan todos aquellos seres vivos que disponen de un sistema nervioso. Es una experiencia asociada a una lesión tisular o como si esta existiera. Su clasificación según el tiempo de evolución; dolor agudo y crónico. Según la fisiología 
del dolor; dolor nociceptivo y neurópatico. En 1965 Melzack y Wall propusieron la teoría de la Compuerta o Puerta de Entrada para explicar los fenómenos relacionados con el dolor. La teoría de la compuerta del dolor es una explicación de cómo la mente desempeña un papel esencial en la opinión del dolor.

La fisiopatología del dolor músculo esquelético considera implicados la inflamación, la fibrosis y la degradación del tejido; refiriéndose al dolor traumático, hombro doloroso, cervicalgia, lumbalgias, síndrome del túnel carpiano, dedos gatillo, tendinitis, luxaciones (11).

Los Trastornos Músculo-Esqueléticos (TME) causados por traumatismo acumulativo, son lesiones o daños a los tejidos corporales que se han ido desarrollando con el paso del tiempo por diversas fuerzas externas, que afectan primariamente a los músculos, tendones, nervios y vasos sanguíneos, y que incluyen una gran variedad de lesiones y enfermedades que resultan de exposiciones repetidas o durante largo tiempo a estrés físico. Los efectos están regularmente relacionados con posturas corporales, energía de movimiento o fuerzas extensoras, así como la duración o repetitividad. Los TME se encuentran entre los problemas más importantes de salud en el trabajo, tanto en los países desarrollados como en los que están en vías de desarrollo. Se cree que la proporción de las enfermedades músculo-esqueléticas atribuibles al trabajo es de alrededor del 30\%; producen molestias o dolor local y restricción de la movilidad, que pueden obstaculizar el rendimiento normal en el trabajo o en otras tareas de la vida diaria (12).

La postura de sentado confiere una disposición especial a la columna vertebral, que se adapta a las situaciones a las que se ve forzada, creando curvas de compensación; cuando el umbral de tolerancia se ve sobrepasado, los sistemas de reequilibrio fracasan y aparece el dolor, que mantenido en el tiempo, agravará la presencia de lesiones simples de contractura muscular en lesiones claras articulares, que dificultará el desarrollo del ejercicio profesional. Estos dolores se localizan con frecuencia en cuello, espalda, hombros, codos, muñecas y manos. En la actualidad en el Instituto Nacional de Salud y Seguridad Ocupacional (NIOSH) establece que los desórdenes músculo-esqueléticas, si han sido causados o agravados por las condiciones y/o medio ambiente de trabajo se les denomina Lesiones Músculo Esqueléticas Ocupacionales (LMEO) (1314).
La frecuencia de dolor musculoesquelético y cuáles son las zonas más frecuente de dolor, con la finalidad de contribuir a la fomentación de una cultura de medidas preventivas para los riesgos laborales y de esta manera a la disminuir el dolor debido a las lesiones músculo esqueléticas, con el fin de que en un futuro se pueda ejecutar un desarrollo laboral con eficacia y efectividad sin ninguna lesión en el desarrollo profesional del terapeuta.

Vernaza y Paz identificaron la frecuencia y los factores de riesgo de dolor músculo-esquelético en los fisioterapeutas asistenciales que laboran en la ciudad de Popayán Colombia. Se realizó un estudio observacional descriptivo a 27 Fisioterapeutas asistenciales; se obtuvo como resultado que el 63,0 $\%$ de los fisioterapeutas presentaron dolor músculoesquelético en los últimos 6 meses. Las áreas afectadas fueron el cuello y zona baja de la espalda seguida de la zona media de la espalda y en menor proporción en $\operatorname{codos}(2)$.

Leyva et al., determinaron el riesgo ergonómico laboral de 9 fisioterapeutas del Departamento de Investigación y Docencia de Lesiones Centrales del Instituto Nacional de Rehabilitación (INR) en el Callao - Perú. Para ello utilizó la Rapid Entire Body Assessment (REBA), permitiendo el análisis de las posiciones adoptadas por los miembros superiores, tronco, cuello y los miembros inferiores de los fisioterapeutas. Cada fisioterapeuta fue evaluado durante su labor con 3 pacientes en 5 posturas diferentes: posición de 4 puntos, arrodillado con apoyo anterior, sentado, bípedo y posición de paso anterior. En 7/9 fisioterapeutas se encontraron niveles de riesgo alto y en 2/9 riesgo medio, así también $5 / 5$ mujeres y $2 / 4$ varones tuvieron riesgo alto y $2 / 4$ varones tuvieron riesgo medio (3).

Maco halló la prevalencia del dolor músculoesquelético ocupacional en los alumnos de postgrado de la Facultad de Odontología de la Universidad Nacional Mayor de San Marcos, de 78 participantes el $87,2 \%$ presentó dolor músculo-esquelético, con respecto al sexo las mujeres presentaron relativamente mayor percepción de dolor, los años de ejercicio profesional y las horas de jornada laborales son directamente proporcionales al dolor. Se encontró mayor prevalencia de percepción de dolor en cuello $71,8 \%$, seguido por zona lumbar $64,1 \%$ y la zona dorsal $53,8 \%$, las actividades clínicas predominantes en la profesión con mayor percepción de dolor fueron las de Endodoncia y Rehabilitación oral-Operatoria 
dental y la intensidad más prevalente de dolor musculo-esquelético percibido por la población fue la intensidad moderada (4).

Cromie et al., investigaron la prevalencia y severidad de los trastornos músculo-esqueléticos de los fisioterapeutas, indagar cuáles son los factores de riesgo contribuyentes y cuáles son sus respuestas a las lesiones (15), se tomó una muestra de 536 terapeutas físicos del estado de Victoria en Australia y se les distribuyó un cuestionario el cual fue enviado por correo electrónico. Se obtuvo como resultado que la prevalencia de trastornos músculo-esqueléticos en los últimos 12 meses fue de $82,8 \%$. La región lumbar fue la más afectada $(62,5 \%)$, seguida del cuello $(47,6 \%)$, región dorsal $(41 \%)$, pulgares $(33,6)$, hombro $(22,9)$, muñeca-mano, codos, rodillas, caderas y pie. Se mostró que los fisioterapeutas más jóvenes reportaron más síntomas en la región lumbar, cervical, dorsal y pulgar en comparación con los fisioterapeutas mayores. El dolor de rodilla se relacionó con el aumento de la edad. No se encontraron diferencias significativas entre terapeutas hombre y mujeres. Los terapeutas que habían trabajado en fisioterapia deportiva o pediatría tenían más probabilidad de generar un trastorno músculo-esquelético. La estrategia de prevención más utilizada fue ajustar la altura de la camilla y/o superficie de apoyo antes de tratar a un paciente (88\%), seguida por trabajar en posturas cómodas y no fatigantes $(86,1 \%)$ y pedir ayuda a alguien en el manejo de pacientes dependientes $(64,7 \%)$ y la menos utilizada fue realizar estiramientos antes de comenzar la jornada laboral o antes de la realización de determinadas técnicas como las manuales $(79,5 \%$ dijo hacerlo casi nunca). (15)

Rodriguez encontró que la prevalencia de lesiones en los fisioterapeutas, y relacionarlas con factores epidemiológicos como sexo, edad, años en actividad y horas de trabajo semanales; encontrar con qué frecuenciallevanacabolosfisioterapeutas determinadas medidas ergonómicas; conocer qué factores de riesgo consideran más influyentes en el desarrollo de dichas lesiones; y averiguar con qué frecuencia llevan a cabo determinadas medidas preventivas (16), se tomó una muestra de 68 fisioterapeutas y se administró un cuestionario. Se obtuvo como resultados que el 60,3\% de los participantes sufrieron alguna lesión músculoesquelética relacionada con el trabajo en los últimos 12 meses. La principal localización anatómica en la que se encontró una mayor frecuencia de lesiones musculoesqueléticas fue de la columna cervical (52,9\%), seguida de la columna lumbar (45,5\%). La principal medida ergonómica fue la regulación de la altura de la camilla y el mantenimiento del centro de gravedad cercano al cuerpo. El factor de riesgo considerado más influyente fue el mantenimiento de posturas incómodas y poco fisiológicas. La principal medida preventiva llevada a cabo por los fisioterapeutas fue "Trabajar en posturas cómodas y no fatigantes"(16).

El objetivo del presente trabajo fue determinar la frecuencia del dolor musculoesquelético en Fisioterapeutas Pediátricos en instituciones de salud de la ciudad de Lima. Describir la presencia de dolor en los últimos 12 meses en Fisioterapeutas Pediátricos en instituciones de salud de la ciudad de Lima. Describir las zonas de dolor musculoesqueléticos en Fisioterapeutas Pediátricos en instituciones de salud de la ciudad de Lima. Describir las medidas preventivas en los Fisioterapeutas Pediátricos en instituciones de salud de la ciudad de Lima.

\section{MATERIAL Y MÉTODOS}

El presente estudio es de tipo observacional descriptivo de corte transversal (18). Se llevó a cabo en Fisioterapeutas pediátricos; en el departamento de Lima teniendo como sedes para realizar la investigación a la Clínica Hogar San Juan de Dios con 29 fisioterapeutas pediátricos, Instituto para el Desarrollo Infantil - ARIE con 18 fisioterapeutas pediátricos; que se encuentran laborando en la actualidad. Y como criterio de inclusión se tomó en cuenta a los fisioterapeutas pediátricos que laboren en las dos instituciones, hombres y mujeres y que deseen participar del estudio. Los criterios de exclusión se hicieron en fisioterapeutas

que se hayan lesionado fuera de su campo laboral o que no quieran ser parte del estudio.

\section{Procedimientos para solicitud de permisos}

Se presentó el proyecto a la Facultad de Medicina de la Universidad Peruana Cayetano Heredia (UPCH) para su evaluación y aprobación. Posterior a su aprobación, nos dirigimos a cada institución con nuestra carta de presentación, solicitando los permisos correspondientes a las autoridades de dichas sedes (director, comité de ética, Jefe de la Unidad de Capacitación, Docencia e Investigación).

El instrumento utilizado fue un cuestionario sobre dolor músculo esquelético en fisioterapeutas pediátricos, elaborada por los autores del estudio, y ayuda de nuestros asesores, usando como referencia el cuestionario Nórdico. Para hacer valido el instrumento 
se eligió a 3 docentes expertos teniendo en cuenta su experiencia, grado académico y disponibilidad, y así pudieran valorar cada uno de los ítems en claridad, redacción, pertinencia de la pregunta y finalmente alguna observación o comentario con respecto al cuestionario en general. El instrumento construido presentó un alto valor con el coeficiente $\mathrm{V}$ de Aiken entre 0,7 y 0,8 que permite establecer el valor de contenido de las variables a ser estudiadas en el cuestionario.

Posterior a la aprobación de parte las instituciones donde se desarrolló el estudio, se inició una visita previa a cada área para entrevistarse con cada terapeuta explicándole la finalidad del estudio, coordinamos horarios disponibles para el llenado del cuestionario, las cuales se distribuyeron de la siguiente manera: tres fisioterapeutas diarios por cinco días a la semana, después de su jornada laboral y dentro de su misma área de trabajo, previamente antes de aplicar el cuestionario los participantes firmaron un consentimiento informado, aceptando su participación en el estudio.

La información fue captada por las investigadoras previamente entrenadas, luego pasó por los procesos de codificación y consistencia previamente al ingreso en una matriz de datos. Luego se elaboró una matriz de datos para el ingreso de datos en el programa Excel. Para realizar el control de calidad de los datos ingresados a la matriz, se revisó aleatoriamente las 5 fichas, el $10 \%$ de datos ingresados, encontrándose error en el ingreso de dos datos por lo cual se procedió a la revisión de todos los datos ingresados.

El estudio es un diseño descriptivo, por lo que se emplea la estadística descriptiva. La variable principal de estudio dolor, zonas de dolor músculo-esquelético y estrategias de afrontamiento se presentan en tablas con frecuencias absolutas y relativas. Las otras variables categóricas: sexo, institución donde labora, área de desempeño y hábitos deportivos también se presentan en tablas con frecuencias absolutas y relativas. A las variables numéricas: edad, y años de experiencia se presentan con los estadísticos de tendencia central y de dispersión. El procesamiento se realizó en el programa estadístico Stata versión 13.

El presente estudio se presentó a evaluación en el Comité Institucional de Ética para Humanos CIE de la Universidad Cayetano Heredia como revisión rápida.

Los resultados se almacenaron en una base de datos dentro de un computador donde está protegido por una clave que solo lo saben los autores de la investigación

\section{RESULTADOS}

El grupo de estudio estuvo conformado por 47 fisioterapeutas pediátricos de los cuales 38 $(80,9 \%)$ fueron de sexo femenino y $9(19,2 \%)$ de sexo masculino, se encontró que el $50 \%$ de los fisioterapeutas pediátricos tuvieron 28 años a menos, mientras el $75 \%$ de los fisioterapeutas tuvieron 32 años a menos. Además, se encontró que la edad del $50 \%$ del sexo femenino fue también 28 años, y para el sexo masculino fue de 34 años. Con respecto al 75\% el sexo femenino fue 29 años, y para el sexo masculino 42 años (tabla 1).

Se observa que el $50 \%$ de los fisioterapeutas pediátricos tuvieron 4 años de experiencia en el área de pediatría a menos, mientras el $75 \%$ de los fisioterapeutas tuvieron 8 años a menos. Además, se encontró que los años de experiencia del $50 \%$ del sexo femenino fueron 3,6 años, y para el sexo masculino 11 años. Con respecto al $75 \%$ el sexo femenino fue 5 años, y para el sexo masculino 15 años (tabla 2).

En cuanto a las horas de trabajo desempeñadas por fisioterapeutas pediátricos en las instituciones de salud el $89,4 \%$ trabaja entre 6 a 8 horas, que al separarlo por sexo se observa que el $92,1 \%$ de las mujeres trabaja entre 6 a 8 horas, mientras que en los hombres solo es el $77,8 \%$. Además, que el género que trabaja entre 9 a 10 horas es el masculino con el 22,2\% (tabla 3 ).

Tabla 1. Edad de los fisioterapeutas pediátricos que presentaron dolor musculoesqueletico, según sexo.

\begin{tabular}{lrcccccc}
\hline Sexo & Media & Desviación estándar & Min & $\mathbf{p}^{\mathbf{2 5}}$ & $\mathbf{p}^{\mathbf{5 0}}$ & $\mathbf{p}^{\mathbf{7 5}}$ & $\mathbf{M a x}$ \\
\hline Femenino & 28,9 & 4,9 & 23 & 26 & 28 & 29 & 45 \\
Masculino & 36,2 & 10,8 & 23 & 26 & 34 & 42 & 53 \\
Total & 30,3 & 6,9 & 23 & 26 & 28 & 32 & 53 \\
\hline
\end{tabular}


Tabla 2. Años de experiencia en el área pediátrica de los fisioterapeutas pediátricos, según sexo.

\begin{tabular}{lccccccc}
\hline Sexo & Media & Desviación estándar & Min & $\mathbf{p}^{\mathbf{2 5}}$ & $\mathbf{p}^{\mathbf{5 0}}$ & $\mathbf{p}^{\mathbf{7 5}}$ & $\mathbf{M a x}$ \\
\hline Femenino & 4,8 & 4,2 & 1 & 2,6 & 3,6 & 5 & 20 \\
Masculino & 11,3 & 8,0 & 1 & 4 & 11 & 15 & 24 \\
Total & 6,1 & 5,7 & 1 & 3 & 4 & 8 & 24 \\
\hline
\end{tabular}

Tabla 3. Horas de trabajo de los fisioterapeutas pediátricos, según sexo.

\begin{tabular}{lcccccc}
\hline $\begin{array}{c}\text { Horas de } \\
\text { Trabajo }\end{array}$ & $\begin{array}{c}\text { Femenino } \\
\text { N=38 }\end{array}$ & $\mathbf{1 0 0 \%}$ & Masculino & $\mathbf{1 0 0 \%}$ & $\begin{array}{c}\text { Total } \\
\mathbf{N}=\mathbf{4 7}\end{array}$ & $\mathbf{1 0 0 \%}$ \\
\hline $6-8$ & 35 & 92,1 & 7 & 77,8 & 42 & 89,4 \\
$9-10$ & 3 & 7,9 & 2 & 22,2 & 5 & 10,6 \\
\hline
\end{tabular}

Tabla 4. Características de la institución, área de desempeño y hábitos deportivos.

\begin{tabular}{lcc}
\hline Características & $\mathbf{n = 4 7}$ & $\mathbf{1 0 0 \%}$ \\
\hline Institución donde labora & & \\
Privada & 18 & 38,3 \\
Pública & 29 & 61,7 \\
Área de Desempeño & & \\
Intervención temprana & 2 & 4,3 \\
Misceláneas & 1 & 2,1 \\
neurología & 11 & 23,4 \\
Ortopedia & 17 & 36,2 \\
Psicomotricidad & 4 & 8,5 \\
Reeducación Motriz & 11 & 23,4 \\
Reeducación Neurológica & 1 & 2,1 \\
Hábitos Deportivos & & \\
Nunca & 7 & 14,9 \\
A veces & 29 & 61,7 \\
Siempre & 11 & 23,4 \\
\hline
\end{tabular}

Considerando la institución donde labora los fisioterapeutas pediátricos se encuentra que el $61,7 \%(29 / 47)$ trabaja en una institución pública y el $38,3 \%(18 / 47)$ en una institución privada. Respecto al área de desempeño, el 36,2\%(17/47) de fisioterapeutas encuestados desempeñan en el área de Ortopedia, seguida de Reeducación Motriz y Neurología con un $23,4 \%$. Por otro lado, referente a los hábitos deportivos el 61,7\%(29/47) de los fisioterapeutas encuestados a veces realizan una actividad deportiva (tabla 4 ).
Se muestra las zonas del dolor musculoesqueléticos referido por los fisioterapeutas $(n=47)$, el $78,7 \%$ de los fisioterapeutas pediátricos refieren que han tenido dolor en cuello, dolor de la espalda superior con $72,3 \%$ y en menor porcentaje dolor en codos con $4,3 \%$. El dolor referido de los fisioterapeutas en los últimos 12 meses, el área del cuello es la zona más frecuente donde se encuentra dolor musculoesqueléticos con el $72,3 \%$, seguida de la espalda superior con $68,1 \%$ y con menor presencia en el área de los codos con un 4,3\%. Con respecto a la presencia de dolor en los últimos 
Tabla 5. Localización y presencia del dolor musculoesqueléticos en fisioterapeutas pediátricos en los últimos 12 meses.

\begin{tabular}{|c|c|c|c|c|c|c|c|c|}
\hline \multirow[t]{2}{*}{ Área } & \multicolumn{2}{|c|}{$\begin{array}{l}\text { Ha tenido } \\
\text { dolor }\end{array}$} & \multicolumn{2}{|c|}{$\begin{array}{l}\text { Ha tenido dolor } \\
\text { en los últimos } \\
12 \text { meses }\end{array}$} & \multicolumn{2}{|c|}{$\begin{array}{l}\text { Ha tenido } \\
\text { dolor en los } \\
\text { últimos } 7 \text { días }\end{array}$} & \multicolumn{2}{|c|}{$\begin{array}{l}\text { Ha recibido } \\
\text { tratamiento } \\
\text { en los últimos } \\
12 \text { meses }\end{array}$} \\
\hline & $\mathrm{n}=47$ & $\%$ & $\mathrm{n}=47$ & $\%$ & $\mathrm{n}=47$ & $\%$ & $\mathrm{n}=47$ & $\%$ \\
\hline Cuello & 37 & 78,7 & 34 & 72,3 & 23 & 48,9 & 7 & 14,9 \\
\hline Hombros & 30 & 63,8 & 24 & 51,1 & 14 & 29,8 & 1 & 2,1 \\
\hline Espalda superiro & 34 & 72,3 & 32 & 68,1 & 17 & 36,2 & 7 & 14,9 \\
\hline Codos & 2 & 4,3 & 2 & 4,3 & 2 & 4,3 & 0 & 0 \\
\hline Muñecas / manos & 33 & 70,2 & 29 & 61,7 & 15 & 31,9 & 7 & 14,9 \\
\hline Espalda baja & 30 & 63,8 & 29 & 61,7 & 12 & 25,5 & 5 & 10,6 \\
\hline Caderas / muslo & 12 & 25,5 & 10 & 21,3 & 5 & 10,6 & 1 & 2,1 \\
\hline Rodillas & 20 & 42,6 & 16 & 34,0 & 9 & 19,2 & 1 & 2,1 \\
\hline Tobillos / pies & 11 & 23,4 & 9 & 19,2 & 7 & 14,9 & 2 & 4,3 \\
\hline
\end{tabular}

Tabla 6. Presencia de dolor en fisioterapeutas pediátricos en los últimos 12 meses según zonas de dolor.

\begin{tabular}{|c|c|c|}
\hline & $n=47$ & $100 \%$ \\
\hline \multicolumn{3}{|c|}{ Ha tenido dolor } \\
\hline Sin dolor & 0 & 0.0 \\
\hline Con dolor & 47 & 100,0 \\
\hline 1 a 2 zonas & 9 & 19,1 \\
\hline 3 a 4 zonas & 14 & 29,8 \\
\hline 4 a 6 zonas & 17 & 36,2 \\
\hline 7 a 8 zonas & 7 & 14,9 \\
\hline \multicolumn{3}{|c|}{ Dolor los últimos 12 meses } \\
\hline Sin dolor & 2 & 4,3 \\
\hline Con dolor & 45 & 95,7 \\
\hline 1 a 2 zonas & 8 & 17 \\
\hline 3 a 4 zonas & 16 & 34 \\
\hline 4 a 6 zonas & 21 & 44,7 \\
\hline 7 a 8 zonas & 0 & 0,0 \\
\hline \multicolumn{3}{|c|}{ Dolor los últimos 7 días } \\
\hline Sin dolor & 5 & 10,6 \\
\hline Con dolor & 42 & 89,4 \\
\hline 1 a 2 zonas & 18 & 38,3 \\
\hline 3 a 4 zonas & 18 & 38,3 \\
\hline 4 a 6 zonas & 6 & 12,8 \\
\hline 7 a 8 zonas & 0 & 0,0 \\
\hline
\end{tabular}

7 días con $48,9 \%$ fue el área del cuello, en segundo orden fue la espalda superior con $36,2 \%$ y con menos presencia en los codos con 4,3\%. Así mismo el área que ha recibido tratamiento en los últimos 12 meses fue en el cuello con un $14,9 \%$ y en espalda superior 14,9 (tabla 5).

La presencia de dolor en fisioterapeutas pediátricos según zonas, estos dolores abarcaban desde 1 a 8 zonas de dolor músculo esqueléticas. El estudio reportó que el 100\% (47) fisioterapeutas pediátricos presentaron dolor alguna vez, con mayor cantidad de zonas con dolor de 5 a 6 . La presencia de dolor los últimos 12 meses fue $95,7 \%$ (45/2) teniendo la mayor cantidad de zonas con dolor de 5 a 7 . Con la presencia de dolor en los últimos 7 días fue $89,4 \%$ (42/5), teniendo la mayor cantidad de zonas con dolor de 1 a 4 (tabla 6).

Se describen las diferentes estrategias de afrontamiento que usan los fisioterapeutas pediátricos en su área laboral. Se observa que los fisioterapeutas pediátricos algunas veces suelen pedir ayuda en el manejo de pacientes dependientes con 46,8\% (22/47), habitualmente trabajan en posturas cómodas y no fatigantes $48,9 \%$ (23/47), aunque casi nunca realizan estiramiento antes de la jornada laboral 46,8\% (22/47), algunas veces pueden realizar descansos o pausas $48,9 \%(23 / 47)$ realizan cambios de posturas con regularidad 38,3\% (18/47) y siempre utilizan diferentes partes del cuerpo para ejecutar una técnica fisioterapéutica 48,9\% (23/47) (tabla 7).

\section{DISCUSIÓN}

Los resultados de este estudio muestran que nuestra población estuvo conformada en su mayoría por mujeres $80,85 \%$. Por otro lado la edad promedio fue de 30,3 años y el promedio de años de experiencia 
Tabla 7. Estrategias de afrontamiento de los

fisioterapeutas pediátricos

\begin{tabular}{lcc}
\hline ESTRATEGIAS & $\mathbf{n}=\mathbf{4 7}$ & $\mathbf{1 0 0 \%}$ \\
\hline Pide ayuda en el manejo de pacientes & dependientes \\
Casi nunca & 17 & 36,2 \\
Algunas veces & 22 & 46,8 \\
Habitualmente & 5 & 10,6 \\
Siempre & 3 & 6,4 \\
Trabaja en posturas cómodad y no fatigantes \\
Casi nunca & 2 & 4,3 \\
Algunas veces & 11 & 23,4 \\
Habitualmente & 23 & 48,9 \\
Siempre & 11 & 23,4 \\
Realiza estiramientos antes de la jornada laboral o \\
de determinadas técnicas & \multicolumn{3}{|c}{} \\
Casi nunca & 22 & 46,8 \\
Algunas veces & 18 & 38,3 \\
Habitualmente & 5 & 10,6 \\
Siempre & 2 & 4,3
\end{tabular}

Realiza descansos o pausas en la jornada laboral

\begin{tabular}{lcc} 
Casi nunca & 16 & 34,0 \\
Algunas veces & 23 & 48,9 \\
Habitualmente & 6 & 12,8 \\
Siempre & 2 & 4,3 \\
Realiza cambios de postura con regularidad & \\
Casi nunca & 2 & 4,3 \\
Algunas veces & 10 & 21,3 \\
Habitualmente & 18 & 38,3 \\
Siempre & 17 & 36,2 \\
Utiliza diferentes partes del cuerpo para ejecutar \\
una técnica fisioterapéutica & \multicolumn{3}{c}{} \\
Algunas veces & 3 & 6,4 \\
Habitualmente & 21 & 44,7 \\
Siempre & 23 & 48,9 \\
\hline
\end{tabular}

en el área pediátrica es de 6,1 años. Dicho resultados son similares al estudio realizado en Colombia (2) encontrando como resultados en su mayoría en mujeres con un $88,9 \%$, edad promedio de 31,2 años y el promedio de tiempo profesional en 7,9 años. Con respecto a la localización anatómica de dolor músculo-esqueléticas, este estudio encontró que la zona más frecuente se presentó en el área cervical, el $72,3 \%$ de los fisioterapeutas pediátricos refirieron haber presentado dolor musculoesqueléticos en los últimos 12 meses en esta zona corporal, seguida por la espalda superior $(68,1 \%)$. En la investigación realizada por Vernaza (2) y en Australia (15), muestran un panorama diferente ya que la región lumbar fue la más afectada seguida de la región cervical. En la investigación elaborada en España (16) se encontró mayor frecuencia en la zona cervical $(52,9 \%)$, seguida por la columna lumbar $(45,5 \%)$. De lo mencionado en las investigaciones anteriores, se puede observar que los fisioterapeutas pediátricos de este estudio están principalmente expuestos a adoptar posturas forzadas de tronco (rotaciones e inclinaciones) y de mantener posturas prolongadas o estar mucho tiempo de pie, y a las manipulaciones repetitivas de cargas.

Lo que compete a la aplicación de las estrategias de prevención, el estudio encontró que la estrategia que casi nunca es aplicada por los fisioterapeutas es realizar estiramiento antes de comenzar la jornada laboral o antes de la realización de determinadas técnicas pues el $46.8 \%$ dijo casi nunca realizarlo, estas cifras son un poco más de la mitad a la encontrada en la investigación en España (16) que fue $79,4 \%$ pero semejante en Australia (15) donde el 79,5\% dijo casi nunca también. Estos datos resultan sumamente importantes ya que dichas estrategias se les enseña a sus pacientes para evitar riesgo de sufrir alguna lesión futura, pero según vemos los fisioterapeutas pediátricos son los que presentan dolor a pesar de tener el conocimiento del buen manejo de técnicas de estiramiento muscular, que no lo practican. Por otro lado el $48,9 \%$ de los fisioterapeutas pediátricos menciono solo algunas veces tener descanso o pausas en su jornada laboral, mientras que en el estudio realizado en la Universidad Mayor de San Marcos (17) supera con un $50 \%$ en algunas veces llevar a cabo esta estrategia. Cabe mencionar que los fisioterapeutas en su mayoría realizan en muy pocas ocasiones estrategias de prevención en su jornada laboral.

La mayor limitación del presente estudio fue la falta de accesibilidad a otras instituciones para ejecutar el proyecto de investigación, ya que el trámite administrativo era tedioso y exigían que uno de nuestros asesores tuviera que ser de su institución por lo que no se ingresó a dos instituciones de salud.

Los resultados de la presente investigación no pueden ser generalizados a todos los fisioterapeutas pediátricos de las instituciones pública o privadas por haber seleccionado a conveniencia la participación de los sujetos de estudio (muestreo no probabilístico).

En este estudio por ser de tipo descriptivo no establece relación causal entre dolor y el desempeño 
laboral, solo describe la presencia de dolor musculoesquelético en este grupo de profesionales.

\section{CONCLUSIONES}

El $100 \%$ de los fisioterapeutas encuestados ha presentado dolor en algún momento y al menos una zona o área del cuerpo; en los últimos 12 meses el 95,7 $\%$ y en los últimos 7 días el $89,4 \%$.

Con respecto al sexo y edad se halló que el sexo femenino predominó mayor proporción $(80,85 \%)$ en comparación con el sexo masculino $(19,15 \%)$.

La zona anatómica más frecuente a padecer dolor es la zona del cuello con un 72,3\% y a la vez es la zona más susceptible a presentar futuras lesiones músculo esqueléticas como también es la zona de la espalda superior $68,1 \%$, seguidamente de la zona de muñeca/ manos y espalda baja con un $61,7 \%$.

Las medidas preventivas que los fisioterapeutas pediátricos realizan en su jornada laboral es la de utilizar diferentes partes del cuerpo para ejecutar una técnica fisioterapéutica; sin embargo, no todos realizan estiramientos antes de la jornada laboral o de determinadas técnicas.

\section{Se recomienda:}

Elaborar un plan de prevención para evitar lesiones musculoesqueléticos en fisioterapeutas pediátricos empezando por la incorporación de cursos desde pregrado y capacitando a fisioterapeutas que laboran en los diversos centros de salud.

Alternar tareas donde incorpore diferentes posiciones corporales para reducir la frecuencia de movimientos repetitivos y evitar posturas mantenidas, o apoyo de un tercero durante el desarrollo del tratamiento.

No llegar a los límites de cargas permitidos (25 $\mathrm{kg}$ en varones y $15 \mathrm{~kg}$ en mujeres). se podría pedir el apoyo del paciente pero en este caso con fisioterapeutas pediátricos no se puede fomentar dicha colaboración, sin embargo se puede utilizar ayudas mecánicas como barras de apoyo para ayudar a la movilización y/o traslado.

Proponer a las instituciones de salud donde laboran, un programa o taller dirigido de estiramientos musculares de manera que el fisioterapeuta lo realice antes de iniciar la jornada laboral y eso ayudará a mejorar su flexibilidad.

Realizar trabajos continuos de 1 o 2 horas deberían realizar pausas durante la jornada

laboral min de 15 minutos.

\section{Correspondencia}

Lucía Oropeza Melgar

Correo electrónico: lucia.oropeza.m@upch.pe

\section{REFERENCIAS BIBLIOGRAFÍCAS}

1. Organización Mundial de la Salud. Comité de Expertos de la OMS en Rehabilitación. Médica. Ginebra: Organización Mundial de la Salud; 1968.

2. Vernaza P, Paz C. Dolor Músculo-Esquelético en Fisioterapeutas del Municipio de Popayán. Tesis de grado. Colombia: Universidad del Cauca;2006.

3. Leyva B, Martínez J, Meza J, Martínez A, Cernaqué C. Riesgo ergonómico laboral en fisioterapeutas de un centro de rehabilitación física. Rev Med Hered. 2011; 22(1):42-43.

4. Maco M. Dolor musculoesquelético ocupacional en alumnos de postgrado de la Facultad de Odontología de la Universidad Nacional Mayor de San Marcos. Tesis para optar el título de Cirujano Dentista. Lima: Universidad Nacional Mayor de San Marcos; 2009.

5. Gutiérrez M, Flores C, Monzó J. Prevalencia de Trastornos Músculo-Esqueléticos de Columna Lumbar en Trabajadores y Límites Biomecánicas en el Manejo de Carga y Pacientes. Rev Cienc Trab. 2010; 12 (37): 380-385.

6. Instituo Nacional de Seguridad e Higiene en el Trabajo. Guía Técnica para la evaluación y prevención de los riesgos relativos a la manipulación manual de cargas. Prevención Trabajo y salud. Rev Inst Nac Seg e hig trabajo. 2004; 2:31.

7. Jimenez U. Lumbalgia ocupacional y discapacidad laboral. Rev fisio España. 2007;6 (2): 17-26.

8. Arteaga A, Ibáñez J, Campos T, Gilli M, Pérez C, García G. Valoración de factores de riesgo del dolor lumbar mecánico en el personal de salud. Rev Esp de Rehab. 1995;29(2):118-122.

9. American Academy of Orthopedic Surgeons. Musculoskeletal conditions in the United States. Park Ridge: AAOS; 1992.

10. Alvarado C. Lesiones musculoesqueléticas. generalidades. seminario de especialización en salud ocupacional. Caracas: Universidad Central de Venezuela;2003.

11. Cailliet R. Incapacidad y dolor de tejidos blandos. Ciudad de México: Editorial El Manual Moderno; 1997. 
12. Riihimäki H, Viikari E. Sistema Músculo-esquelético. Oficina Internacional del Trabajo. Enciclopedia de Salud y Seguridad en el Trabajo. Ginebra: Oficina Internacional del Trabajo; 1995. p. 1-39.

13. Centros paraelcontrolylaprevencióndeenfermedades. Ergonomía (desordenes musculoesqueléticos). Georgia: Instituto Nacional para la seguridad y salud ocupacional (NIOSH); 2012. (Citado el 21 de enero 2015) Disponible en: https://www.cdc.gov/spanish/ niosh/topics/ergonomia.html

14. León N, López A. Lesiones músculo esqueléticas en el personal odontológico. Acta odontol. venez. 2006; 44 (3): 413-418.

15. Cromie J, Valma E, Robertson J, Best M. WorkRelated Musculoskeletal Disorders in Physical Therapists: Prevalence, Severity, Risks, and Responses. Phys Ther. 2000;80(4):336-351.
16. Rodríguez C. Prevalencia de lesiones musculoesqueléticas relacionadas con el trabajo en fisioterapeutas. Tesis para optar el título de Tecnólogo Medico. Alcalá de Henares: Facultad de Fisioterapia, Universidad de Alcalá; 2011.

17. Suárez M. Frecuencia y estrategias de prevención de lesiones músculo-esqueléticas en fisioterapeutas de Lima Metropolitana, diciembre 2012. Tesis para optar el grado el título de Tecnólogo Médico. Lima: Facultad de Medicina Humana. Universidad Nacional Mayor de San Marcos; 2013.

18. Hernández R. Metodología de la investigación. 4ta edición. Ciudad de México: McGraw-Hill;2004.

Recibido: $18 / 01 / 2020$

Aceptado: 10/05/2020 